\title{
EVALUACIÓN DE USABILIDAD EN RECURSOS TIC PARA LA ENSEÑANZA DE METODOLOGÍA DE LA INVESTIGACIÓN A ESTUDIANTES DE PSICOLOGÍA
}

\section{ASSESSMENT OF USABILITY IN TIC RESOURCES FOR THE TEACHING OF RESEARCH} METHODOLOGY TO PSYCHOLOGY STUDENTS

Daniela Jacqueline Vera Solis, Ángel Francisco García Pacheco,

Karina Serrano Alvarado, Mario E. Rojas Russell

Universidad Nacional Autónoma de México, México.

Correspondencia:mario.rojas@zaragoza.unam.mx

\section{RESUMEN}

La formación de la licenciatura en psicología incluye la habilidad de investigación como una de las competencias clave de los futuros profesionales. La Facultad de Estudios Superiores Zaragoza (FESZ) imparte dicha unidad de aprendizaje a lo largo de los ocho semestres que dura la carrera. Previamente, se han descrito experiencias heterogéneas de los estudiantes hacia esta asignatura, en las cuales resalta una falta de afinidad hacia esta materia. Un factor clave son las estrategias de enseñanza utilizadas por los docentes. Se han propuesto a las tecnologías de la información y la comunicación (TICs) como un recurso para complementar esas estrategias. Usando los factores propuestos por Nielsen (1992), se evaluó la usabilidad de ocho recursos basados en TICs para apoyar la enseñanza de la metodología de la investigación (infografías, videos y 
presentaciones en powerpoint). Participaron 163 estudiantes de psicología de la FESZ, de tres semestres diferentes. Los recursos mejor evaluados fueron los videos y las infografías. Las presentaciones en powerpoint fueron los materiales que más recomendaciones de mejora recibieron. Se observaron asociaciones significativas entre el semestre cursado y la experiencia en la asignatura. Se concluye que estos materiales pueden ser útiles en complementar la enseñanza de la metodología de la investigación. Palabras clave: metodología de investigación, enseñanza, recursos TIC, usabilidad.

\section{ABSTRACT}

The training for a bachelor's degree in psychology emphasizes research skills as a critical component of future professionals' competences. This course is taught during the eight semesters of the degree at the Faculty of Higher Studies Zaragoza (FESZ). Previously, varied student experiences with this subject have been documented, highlighting a lack of affinity for the subject. A critical component is the teachers' instructional approach. Information and communications technologies (ICTs) have been proposed as a resource to complement these strategies. Using the factors proposed by Nielsen (1992), the usability of eight ICT-based resources (infographics, videos, and powerpoint presentations) was evaluated to support the teaching of research methodology. 163 FESZ psychology students from three distinct semesters participated. Videos and infographics were the most highly rated resources. PowerPoint presentations received the greatest suggestions for improvement. There were significant correlations between the semester taken and the topic experience. It is concluded that these ICT tools can be helpful in complementing research methodology teaching. Key words: Research methodology, teaching, ICT resources, usability. 


\section{INTRODUCCIÓN}

En la conciencia colectiva la investigación se asocia con los científicos que hacen experimentos en laboratorios; sin embargo, la investigación científica está presente en gran parte de las disciplinas profesionales (Peña, 2014). Una de estas disciplinas es la psicología. En el perfil profesional de las escuelas en las que se imparte esta carrera, se menciona la habilidad de investigación como una de las competencias que se deben tener al egresar de la licenciatura (p. ej. FES Zaragoza, 2010).

La investigación genera conocimiento científico útil para quien lo genera y para quien lo consume. Esto es particularmente relevante para aquellos profesionales responsables de ofrecer servicios a usuarios. Estos, regularmente esperan que el servicio que reciben no solo sea de la mejor calidad, sino sobre todo que sea efectivo en resolver su demanda de atención. Los psicólogos como profesionales prestadores de servicios, están obligados éticamente a prestar servicios basados "en un cuerpo de conocimientos válido y confiable, sustentado en la investigación científica, por lo que el trabajo que desempeña debe corresponder directamente con la educación, formación, experiencia supervisada o experiencia profesional que haya recibido formalmente" (Sociedad Mexicana de Psicología, 2007, p. 47). Una perspectiva de esta naturaleza resalta la formación de los estudiantes de psicología en competencias y habilidades que favorezcan el uso y consumo adecuado de información científica. Sin embargo, se han identificado experiencias de aprendizaje muy heterogéneas de los alumnos de esta licenciatura que no aseguran una adecuada formación en esta perspectiva (Montero, 2019). En relación con las estrategias de enseñanza en los años recientes se ha propuesto que se pueden usar recursos generados con Tecnologías de Información y Comunicación (TIC) como herramientas 
o complementos para el aprendizaje de los contenidos propuestos para la asignatura (Vega, 2016).

En México, de acuerdo con la Encuesta Nacional sobre Disponibilidad y Uso de Tecnologías de la Información en los Hogares (ENDUTIH) en 2016, 65.5 millones de mexicanos contaban con acceso a internet; sin embargo, para el 2019 en esta misma encuesta se reportaron 80.5 millones usuarios de internet, de los cuales el 91.2\% eran jóvenes entre 18 y 24 años de edad y alrededor del $80 \%$ de ellos lo usan para apoyar su educación, es decir buscar información, complementar tareas, etc (INEGI,2020).

\section{La metodología de la investigación}

La investigación se entiende como el proceso de tratar de adquirir, aplicar y crear conocimiento basado en el método científico (Gómez, 2012). En relación con esto, uno de los usos de la metodología de la investigación, además de ser la guía básica para el desarrollo de proyectos de investigación, es una herramienta para interpretar, aplicar o tomar decisiones con base en conocimiento científico a través de criterios estandarizados, por lo que permite que los resultados sean comunicables y aplicables a una población o situación determinada. Estos criterios permiten la replicación de estudios en situaciones o grupos distintos a aquellos en los cuales se desarrolló originalmente una investigación; incluso en algunos casos los conocimientos obtenidos pueden ser útiles para distintas disciplinas (Behar, 2008). Por estas razones, la enseñanza de la metodología de la Investigación forma parte central del currículum de gran parte de las disciplinas que basan su práctica en el conocimiento científico. En las instituciones de educación superior en México la enseñanza de la investigación inició hacia la década de los 1960’s (Sánchez, 2014). 


\section{Importancia de la metodología de la investigación para el desempeño profesional: la práctica basada en la evidencia}

En relación con la oferta académica de la Universidad Nacional Autónoma de México (UNAM, 2018) se observa que la investigación es una competencia curricular constante en múltiples licenciaturas con especial énfasis en las ciencias de la salud y disciplinas relacionadas al trato con usuarios, por ejemplo, la psicología.

La investigación es una competencia requerida no exclusivamente con el propósito de generar futuros investigadores, sino también con el de procurar habilidades necesarias para el desarrollo profesional del psicólogo que le permita el abordaje y la solución de problemas con un enfoque basado en la mejor evidencia científica disponible. Lo cual está orientado, aunque no siempre de manera explícita, con la práctica basada en la evidencia (PBE). Este concepto se refiere a un paradigma de práctica profesional el cual se originó en medicina hacia finales de la década de los 1980 e inicios de los 1990. Sin embargo, tuvo más auge en la primera década de los 2000 y se mantiene hasta la actualidad (Landa, Martínez y Sánchez-Sosa, 2013).

En el mismo sentido, se ha descrito a la práctica de psicología clínica basada en la evidencia como un estándar basado en la investigación con el objetivo de integrar los resultados de investigaciones recientes con la experiencia clínica y las características psicosociales y contextuales de los usuarios para aplicar el mejor tratamiento posible (Quant y Trujillo, 2014). Por ejemplo, David, Cristea y Hofmann (2018), con base en una revisión de investigaciones y ensayos clínicos relacionados señalan a la terapia cognitivo conductual como el estándar de oro para la psicoterapia. 
La PBE se ha orientado en mayor medida hacia el área clínica, pero es un enfoque que se puede realizar sin importar el área en que se desarrolle el psicólogo y, como se describe en los fundamentos para llevar a cabo la PBE, es necesario desarrollar habilidades investigativas para buscar, seleccionar y discriminar información útil que permita una adecuada toma de decisiones en un contexto profesional. No obstante, la percepción de estas habilidades entre estudiantes de psicología es muy variable, lo que sugiere la falta de una formación uniforme en estas competencias. Por ejemplo, en un estudio con poco más de 200 estudiantes de psicología de una universidad del norte de México, Jiménez-Pérez et al. (2020) encontraron que, ante una situación hipotética de solución de un problema profesional, los estudiantes empleaban más tiempo en diseñar una intervención a partir de su propia iniciativa que usando las herramientas de búsqueda y análisis de información científica para encontrar la respuesta a la situación ficticia.

La enseñanza de la metodología de la investigación en las instituciones de educación superior en México inició hacia la década de los 1960s (Sánchez, 2014). En el caso particular de la psicología, su desarrollo se acompañó con el de la perspectiva de la disciplina como una disciplina científica con un enfoque experimental primero, enfocado en el estudio de los procesos psicológicos básicos: aprendizaje, percepción, memoria, etc. y después extendiendo los temas de investigación a los ámbitos de aplicación de la psicología: clínica, educación, organizaciones, etc. De forma concomitante, los planes de estudio incorporaron gradualmente la asignatura de metodología de la investigación en sus planes de estudio (Díaz. 1971).

En el caso de la Carrera de Psicología de la Facultad de Estudios Superiores Zaragoza (FES-Z), su perfil de egreso considera a la metodología de 
la investigación como una competencia importante para promover la mejor formación posible en el campo de la investigación psicológica y la aplicación de resultados mediante el dominio de los conocimientos básicos y la práctica de la profesión. La actividad instruccional del seminario de investigación es el espacio específico dedicado a la enseñanza-aprendizaje de estos contenidos. La actividad se realiza durante los ocho semestres que dura la carrera (FES Zaragoza, 2010).

Aunque se han descrito y propuesto formas de la enseñanza de la psicología en lo general, no parecen existir propuestas específicas para la enseñanza de la metodología de la investigación, con excepción de los procedimientos experimentales.

\section{Experiencias y percepción sobre la enseñanza de metodología de investigación en estudiantes de psicología}

De acuerdo con Ayala, Messing, Labbé y Obando (2010) uno de los puntos claves para evaluar la eficacia de los planes de estudios, es identificar si hay congruencia entre lo planteado y los resultados. En relación con esto, en el período 2018-2019 la Facultad de Estudios Superiores Zaragoza reportó que de los 411 egresados de la carrera de psicología que se titularon en ese periodo, sólo el 21 por ciento $(\mathrm{n}=86)$ lo hicieron por tesis o tesina (Hernández, 2019). Asimismo, el reporte de la Dirección General de Planeación de la UNAM del 2020 informó que para el año 2019, el porcentaje de alumnos titulados por tesis o actividad de investigación fue de 22.4 (20\% y $0.4 \%$, respectivamente) mientras que poco más del $50 \%$ obtuvo su título por la modalidad de Ampliación y Profundización de Conocimientos. Cifras que contrastan con los porcentajes de la Facultad de Psicología y con los de la FES Iztacala, en donde por tesis o actividad de investigación de titulan el $50.7 \%$ y el $39.8 \%$, respectivamente y por ampliación y profundización de 
conocimientos el 43.1\% y 48.6\%, respectivamente (UNAM, 2020). Esto sugiere que las oportunidades de hacer investigación en la FES Zaragoza son notoriamente menores comparadas con las otras dos escuelas. La explicación de estos datos obedece a múltiples factores entre ellos, la poca oferta de proyectos de investigación entre los profesores de la carrera, profesores sin las competencias para dirigir una tesis, poco conocimiento de parte de los estudiantes sobre qué es una tesis y cómo desarrollar una investigación, derivado de una pobre experiencia durante la carrera.

A esto se añaden las dificultades administrativas relacionadas con las características del proyecto y el surgimiento de nuevas modalidades de titulación (Ibarra, 2017). Un reporte cualitativo previo con estudiantes de la carrera de psicología de la FESZ identificó que la valoración de la experiencia (positiva o negativa) con la actividad de seminario de investigación está influenciada directamente por aspectos relacionados con el docente, es decir, que este tenga conocimiento y experiencia previos, ofrezca retroalimentación, elementos motivadores y el estilo o estrategias de enseñanza que implemente para la clase (Montero, 2019).

\section{El papel de las TIC en el proceso enseñanza-aprendizaje}

Respecto a las estrategias de enseñanza, en las últimas décadas se ha generado mucha experiencia acerca del uso de las Tecnologías de la Información y la Comunicación (TIC) para el proceso de enseñanzaaprendizaje ya que se ha demostrado que con estas pueden generarse recursos que apoyen el proceso (López, 2007). El uso de los recursos tecnológicos se ha convertido en una parte relevante de la vida cotidiana, académica y laboral, en consecuencia, ha aumentado la importancia de una buena capacitación de los docentes para generar conocimiento a través de estas tecnologías (Sáenz, 2010). 
El concepto de las TIC se refiere a medios tecnológicos informáticos y telecomunicaciones orientados a favorecer los procesos de información y comunicación. Por otro lado, los recursos TIC son los materiales generados con estas tecnologías, es decir, la aplicación. En el caso de la educación, dichos recursos generan nuevos enfoques en el proceso de enseñanzaaprendizaje, orientados a un cambio estructural de cómo se proporciona la información ya que con ellos se pueden generar áreas de oportunidad para el aprendizaje (Araujo y Bermúdez, 2009).

Entre los beneficios de usar recursos TIC para el aprendizaje, Rodríguez, Mendoza y Ferrer (2014) mencionan que el estudiante puede ser más autónomo y tener un nivel más alto de autorregulación respecto a la forma en la que aprende, debido a que generalmente los recursos se encuentran en línea o en alguna base de datos y en consecuencia el estudiante puede consultarlos cuando los necesite. Además de que puede detenerse a analizarla si lo requiere, para esto es importante que los recursos estén orientados a impulsar al estudiante a la solución de problemas a través de la generación de ideas y conclusiones. No obstante, Araujo y Bermúdez (2009) mencionan algunas limitaciones con relación al uso de este tipo de herramientas en el ámbito educativo: la competencia tecnológica del docente, es decir el manejo adecuado de las TIC para que la implementación de recursos sea efectiva el docente debería manejar los programas y comprender el funcionamiento general de las TIC; la cantidad de recursos tecnológicos (hardware y software) que la institución tiene a disposición tanto de los docentes como de los alumnos.

Cacheiro (2011) clasifica los recursos TIC para la enseñanza en tres grandes grupos: 
- Recursos TIC de información: Permiten obtener datos e información complementaria para abordar un tópico escolar o educativo. Entre los cuales se encuentran webgrafía, enciclopedias virtuales, bases de datos online, etc.

- Recursos TIC de colaboración: Permiten participar en redes de profesionales instituciones, etc. con el objetivo de dialogar o adquirir información sobre alguna temática educativa, en esta categoría se encuentran wikis, blogs, entre otros.

- Recursos TIC para el aprendizaje: Hacen posible el proceso de adquisición de conocimiento, procedimientos y actitudes previstos en el plan de estudios. Esta categoría se refiere a repositorios de recursos educativos, tutoriales, elementos interactivos, cuestionarios online, e-books, podcast, presentaciones, etc.

Por otro lado, existen diversas metodologías a través de las cuales se pueden desarrollar de manera eficiente estos recursos, una de ellas es el diseño instruccional propuesto por Merril (2006) el cual se basa en cinco principios básicos:

1. Centralidad de tareas. Se determina el tema y se busca mostrar el área de oportunidad, la habilidad o los problemas a dominar, en un contexto realista y objetivo.

2. Activación. Se busca estimular el conocimiento previo de la persona, la experiencia es importante para la adquisición de nuevos aprendizajes. 
3. Demostración. El aprendizaje es más factible cuando el conocimiento es observable y puede demostrarse en ese contexto real.

4. Aplicación. La adquisición del conocimiento es más fácil cuando la persona puede poner en práctica las nuevas habilidades y aprendizajes en situaciones reales.

5. Integración. Se facilita el aprendizaje cuando la persona integra el nuevo conocimiento con tareas o problemas habituales en su día a día.

\section{Evaluación de las TIC. La usabilidad de las TIC.}

Sin importar cuál sea el tipo de recurso TIC es importante evaluarlos antes de implementarlos en los planes educativos. Una forma de evaluar los recursos digitales es a través de la usabilidad. Esto se refiere a la eficacia con que los usuarios pueden usar el producto para lograr sus objetivos y qué tan satisfechos están con el proceso (Frege, 2011).

La usabilidad es un concepto que se originó en la informática con el objetivo de evaluar páginas web, aplicaciones o cualquier recurso electrónico para mejorar el software ya sea de forma visual o respecto a su contenido, hasta que el elemento tenga las características necesarias para cumplir su propósito. De acuerdo con Nielsen (1992), las características que un recurso debe cumplir para considerarse usable son: facilidad de aprendizaje, eficiencia de uso, intuitivo y satisfactorio/ útil para el usuario. Para Behrens, Van-Elzakker y Schmidt (2015) un recurso de aprendizaje usable debe: enseñar la información que proporciona, debe ser fácil de recordar; contener solo el material o datos realmente necesarios para el usuario; además debe ser fácil y agradable de ver y usar; finalmente debe ser accesible y fácil de entender para todos los usuarios, incluso si no son 
usuarios frecuentes o no conocen demasiado sobre el tema en cuestión. Respecto a la evaluación sin importar cuál sea el método y técnica que los desarrolladores decidan usar siempre se deben tener en cuenta estos parámetros o atributos de usabilidad.

Con relación con estas estrategias de enseñanza, desde finales de los años 80 's se comenzó a hablar acerca de cómo se pueden implementar las nuevas Tecnologías de Información y Comunicación (TIC) para generar materiales que apoyen el proceso de aprendizaje. Uno de estos materiales y medios son los videos educativos, presentaciones e infografías. Desde investigaciones como la de Pablos-Pons y Cabero-Almenara (1990) se han mostrado resultados favorables hacia este tipo de medios ya que se confirmó que tienen un impacto positivo en el aprendizaje, además se observó que, al apoyar el aprendizaje por medio de los videos, los alumnos eran capaces de comunicar más fácilmente lo aprendido, en comparación con los que obtuvieron aprendizaje de un texto.

Sin embargo, los creadores deben asegurarse de que los recursos sean útiles, es decir que proporcionen la información que los estudiantes necesitan. Asimismo se debe evaluar la usabilidad, es decir la facilidad de acceso a estos materiales. En este contexto esta investigación tuvo como objetivo evaluar la usabilidad de algunos recursos TIC elaborados ex profeso para apoyar el proceso de enseñanza/aprendizaje de contenidos relacionados con la metodología de la investigación dirigidos a estudiantes de psicología de pregrado, bajo un enfoque de práctica basada en evidencia (PBE). El propósito final es que estos recursos puedan ser utilizados tanto por alumnos como por profesores para complementar la enseñanza aprendizaje de los contenidos de esa materia. 


\section{METODO}

\section{Diseño}

Entre los meses de septiembre y noviembre del 2020, se realizó un estudio transversal, de acuerdo con Fernández et al., (2014), los estudios transversales o transeccionales, son aquellos que recolectan datos en un solo momento, en un tiempo único y tienen el propósito de describir variables y analizar su interrelación en un momento dado, por lo que corresponde con el objetivo de este estudio.

\section{Participantes}

Se empleó un muestreo intencional mediante invitaciones electrónicas en la que se solicitó la participación voluntaria en el estudio. Se consideró como único criterio de inclusión estar inscrito en cualquier semestre de la carrera de psicología de la FESZ al momento de la medición. La muestra final estuvo conformada por 163 alumnos distribuidos en cinco semestres: 9 de primer semestre, 67 de tercero, 57 de quinto, 28 de séptimo y 2 de octavo semestre.

\section{Variables e instrumentos}

Recursos TIC para el aprendizaje: Son aquellos que posibilitan la adquisición de conocimientos y procedimientos necesarios para la formación académica, entre ellos se encuentran los repositorios educativos, videos educativos, tutoriales, podcast, etc (Cacheiro, 2011). Dentro de esta investigación se consideraron los siguientes recursos, los cuales estuvieron diseñados con la metodología del diseño instruccional.

- Dos videos tutoriales, en formato mp4: Ambos tutoriales para búsqueda de artículos científicos, el primero en una base de datos Redalyc y el segundo acerca de cómo buscar artículos en Google académico. 
- Cuatro infografías, en formato png: Acerca de los elementos que componen un artículo científico, tales como portada (autores, resumen, palabras clave), introducción, procedimiento y resultados.

- Una infografía interactiva en formato pdf: Con relación a ¿Cómo leer un artículo científico? A través del formato IMRaD.

- Una presentación, en formato ppx: acerca de la discusión de un artículo científico.

Usabilidad: Se refiere a la eficacia con que los usuarios pueden usar el producto para lograr sus objetivos y qué tan satisfechos están con el proceso (Frege, 2011). En esta investigación se evaluó a través de un cuestionario online de usabilidad para recursos TIC diseñado ex profeso el cual se realizó basado en los criterios de Nielsen (1992). El formulario describe el uso se les daría a los datos que proporcionarán los participantes, se hizo énfasis en la confidencialidad, y se proporcionó un correo electrónico de contacto para cualquier duda que pudiera presentarse en los participantes, acerca de cómo contestar o sobre la confidencialidad de sus datos.

\section{Valoración de la experiencia en metodología de la investigación:} Percepción de la experiencia percibida en las clases de metodología de la investigación. Esta variable se midió mediante una escala análoga de 10 unidades en donde el valor mínimo (1) se refería a la peor experiencia y el máximo (10) a la mejor experiencia.

\section{Procedimiento}

El cuestionario se distribuyó a través de redes sociales y correo electrónico entre estudiantes y profesores de la carrera de psicología de la FES Zaragoza. Estuvo disponible durante 14 días para ser contestado. 


\section{Análisis}

Los datos se almacenaron en tiempo real en una hoja de cálculo de google. Se depuró la base de datos, eliminando datos perdidos, inverosímiles o convirtiendo valores en cadena alfanumérica a valores cuantitativos. Se realizó un análisis descriptivo de la muestra con los datos relacionados a los aspectos académicos. Finalmente, se realizó el análisis estadístico con los factores de usabilidad respecto a los recursos TIC para el aprendizaje de la metodología de la investigación. Se realizó la prueba chi-cuadrada de Pearson para identificar la relación de las variables con los aspectos académicos, tales como el semestre en curso, experiencias con la metodología y promedio escolar. Asimismo, se realizó un análisis de varianza no paramétrico con la prueba Kruskal-wallis para contrastar la distribución de la muestra respecto a los factores de usabilidad. Finalmente, se realizó un análisis de correlación de Spearman para identificar la relación entre los factores académicos y la evaluación dada a los factores de usabilidad. Todos los análisis estadísticos se llevaron a cabo en el programa Stata v.13 para Windows.

\section{RESULTADOS}

Participaron 163 alumnos distribuidos en cinco semestres. Dado que del tercero y octavo semestres los participantes fueron solamente nueve y dos, respectivamente, se reunieron en una sola categoría a los estudiantes de tercero con los de primero y a los de séptimo con los de octavo. La Tabla 1 muestra la media de los promedios escolares y la valoración de la experiencia en la actividad de metodología de la investigación por semestre. Se encontraron diferencias significativas en ambas variables entre los tres grupos. En el promedio escolar, el análisis post-hoc mostró que el grupo de primero/tercer semestre tuvo un promedio significativamente menor que los alumnos de quinto y séptimo/octavo, mientras que estos dos últimos 
no fueron significativamente diferentes. A su vez, la valoración de la experiencia de las clases de metodología mostró el mismo patrón: el grupo de primero/tercer semestre fue significativamente diferente del quinto y séptimo/octavo, y estos dos no fueron significativamente distintos entre sí. Llama la atención la disminución de los promedios de las valoraciones conforme avanzan los semestres. Una prueba no paramétrica de tendencia mostró que ésta era significativa $(\mathrm{z}=-.39, \mathrm{p}<.00)$.

Tabla 1

Descripción de la muestra de estudio, $\overline{\mathrm{X}} \pm$ d.e.

\begin{tabular}{|c|c|c|c|c|c|}
\hline 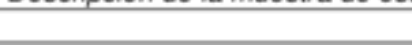 & 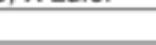 & \multicolumn{4}{|c|}{ Semestres } \\
\hline Variable & $N=163$ & $\begin{array}{c}1^{2} \text { y } 3^{2} \\
n=76\end{array}$ & $\begin{array}{c}5^{2} \\
\mathrm{n}=57\end{array}$ & $\begin{array}{c}7^{2} \text { y } 8^{2} \\
n=30\end{array}$ & p \\
\hline Promedio actual & $8.7 \pm 0.64$ & $8.2 \pm 1.49$ & $9.0 \pm 0.52$ & $8.8 \pm 0.59$ & 0.00 \\
\hline $\begin{array}{l}\text { Evaluación de la experiencia en } \\
\text { metodologia de la investigación }\end{array}$ & $7.89 \pm 1.42$ & $8.3 \pm 1.20$ & $7.6 \pm 1.07$ & $7.2 \pm 2.07$ & 0.00 \\
\hline
\end{tabular}

Respecto a la distribución de los recursos consultados en las infografías, la que atrajo mayor atención fue la revisión del procedimiento de un artículo científico, la cual fue consultada casi ocho veces más que la que se refería a la descripción de la introducción de un reporte. Respecto a los videos el que más atrajo al público fue ¿Cómo utilizar Google académico? que registró más de la mitad de las revisiones (Tabla 2).

Tabla 2

\begin{tabular}{|c|c|c|c|c|c|}
\hline \multirow{2}{*}{\multicolumn{3}{|c|}{$\begin{array}{c}\text { Distribución de la revisión de los recursos } \\
\text { Infografias }\end{array}$}} & \multirow{2}{*}{\multicolumn{3}{|c|}{ Videos }} \\
\hline & & & & & \\
\hline Título & Frecuencia & $\%$ & Título & Frecuencia & $\%$ \\
\hline Formato IMRaD & 36 & 22 & \multirow{3}{*}{$\begin{array}{l}\text { ¿Cómo utilizar Google } \\
\text { académico? }\end{array}$} & \multirow{3}{*}{100} & \multirow{3}{*}{61} \\
\hline Portada & 25 & 15 & & & \\
\hline Introducción & 8 & 5 & & & \\
\hline Unidad de análisis & 31 & 19 & \multirow{3}{*}{$\begin{array}{l}\text { ¿Cómo buscar artículos } \\
\text { científicos? }\end{array}$} & \multirow{2}{*}{63} & \multirow{2}{*}{39} \\
\hline Procedimiento & 63 & 39 & & & \\
\hline $\mathrm{N}$ & 163 & 100 & & 163 & 100 \\
\hline
\end{tabular}


Respecto a la usabilidad, la Tabla 3 presenta los promedios globales de los aspectos evaluados por recurso. Se puede notar que de forma general el video fue el recurso mejor evaluado en todos los aspectos, seguido por la infografía.

Tabla 3

Usabilidad por tipo de recurso ( $\overline{\mathrm{X}} \pm$ d.e.)

\begin{tabular}{lccc}
\hline \multicolumn{1}{c}{ Tipo de recurso } & Infografía & Presentación & Video \\
\hline Facilidad de acceso & $8.71 \pm 0.18$ & $8.72 \pm 0.18$ & $8.92 \pm 0.17$ \\
\hline Atractivo visual del recurso & $7.88 \pm 0.17$ & $6.86 \pm 0.20$ & $8.64 \pm 0.14$ \\
\hline Utilidad de la información & $8.04 \pm 0.16$ & $8.04 \pm 0.18$ & $9.06 \pm 0.14$ \\
\hline Utilidad del recurso como tutorial & $7.87 \pm 0.17$ & $7.74 \pm 0.18$ & $9.03 \pm 0.14$ \\
\hline Probabilidad de futura consulta & $7.26 \pm 0.19$ & $7.29 \pm 0.21$ & $8.63 \pm 0.17$ \\
\hline Probabilidad de recomendar el recurso & $7.63 \pm 0.20$ & $7.23 \pm 0.21$ & $8.71 \pm 0.16$ \\
\hline
\end{tabular}

De manera específica, con relación a la evaluación de la usabilidad de las infografías no se observaron diferencias estadísticamente significativas, en la presentación tampoco se observaron sin embargo a primera vista en los aspectos de Atractivo visual, Utilidad de la información y Utilidad del recurso como tutorial parece haber una tendencia decreciente sobre su usabilidad a medida que avanzan los semestres, sin embargo, ésta no resultó ser estadísticamente significativa.

Finalmente, en lo relacionado a los videos se observa que los alumnos de primero, tercero y quinto semestres calificaron la usabilidad del recurso por encima de la media en cinco de los seis factores. Sin embargo, las diferencias con los participantes de séptimo y octavo semestre no son significativas. Se analizaron posibles tendencias en los factores de atractivo visual del recurso, usabilidad de la información, usabilidad del recurso como tutorial y probabilidad de futura consulta, pero no se encontraron tendencias significativas (Tabla 4). 
Tabla 4

\begin{tabular}{|c|c|c|c|c|c|}
\hline \multicolumn{6}{|c|}{ Usabilidad de los recursos por semestre en curso ( $\bar{x} \pm d . e)}$. \\
\hline Factor de usabilidad & $\mathbf{N}$ & $1^{2}$ y $3^{\circ}$ & $5^{2}$ & $7^{2}$ y $8^{2}$ & $\mathbf{p}$ \\
\hline \multicolumn{6}{|l|}{ Infografia } \\
\hline Facilidad de acceso & $8.72 \pm 2.38$ & $8.57 \pm 2.44$ & $8.96 \pm 2.08$ & $8.6 \pm 2.78$ & 0.17 \\
\hline Atractivo visual & $7.88 \pm 2.23$ & $7.85 \pm 2.05$ & $7.93 \pm 2.30$ & $7.90 \pm 2.60$ & 0.59 \\
\hline Utilidad de la información & $8.04 \pm 2.13$ & $8.06 \pm 1.84$ & $8.05 \pm 2.30$ & $8.0 \pm 2.51$ & 0.18 \\
\hline Utilidad como tutorial & $7.88 \pm 2.30$ & $7.85 \pm 1.88$ & $7.91 \pm 2.55$ & $7.86 \pm 2.77$ & 0.71 \\
\hline Probabilidad de futura consulta & $7.26 \pm 2.51$ & $7.34 \pm 2.24$ & $7.17 \pm 2.68$ & $7.33 \pm 2.90$ & 0.10 \\
\hline Probabilidad de recomendarla & $7.63 \pm 2.66$ & $7.53 \pm 2.34$ & $7.54 \pm 2.96$ & $8.03 \pm 2.87$ & 0.39 \\
\hline \multicolumn{6}{|l|}{ Presentación } \\
\hline Facilidad de acceso & $8.72 \pm 2.30$ & $8.81 \pm 2.12$ & $9.03 \pm 1.93$ & $7.9 \pm 3.15$ & 0.20 \\
\hline Atractivo visual & $6.86 \pm 2.62$ & $7.32 \pm 2.19$ & $6.47 \pm 2.87$ & $6.43 \pm 3.01$ & 0.32 \\
\hline Utilidad de la información & $8.04 \pm 2.29$ & $8.28 \pm 1.86$ & $7.98 \pm 2.43$ & $7.53 \pm 2.94$ & 0.80 \\
\hline Utilidad como tutorial & $7.78 \pm 2.38$ & $8.03 \pm 2.0$ & $7.50 \pm 2.59$ & $7.46 \pm 2.82$ & 0.74 \\
\hline Probabilidad de futura consulta & $7.29 \pm 2.77$ & $7.68 \pm 2.35$ & $7.07 \pm 3.15$ & $6.73 \pm 2.94$ & 0.34 \\
\hline Probabilidad de recomendarla & $7.23 \pm 2.77$ & $7.56 \pm 2.42$ & $6.89 \pm 3.01$ & $7.03 \pm 3.14$ & 0.58 \\
\hline \multicolumn{6}{|l|}{ Videos } \\
\hline Facilidad de acceso & $8.92 \pm 2.25$ & $9.05 \pm 1.91$ & $9.19 \pm 2.00$ & $8.06 \pm 3.20$ & 0.22 \\
\hline Atractivo visual & $8.64 \pm 1.89$ & $8.76 \pm 1.75$ & $8.77 \pm 1.76$ & $8.1 \pm 2.39$ & 0.48 \\
\hline Utilidad de la información & $9.06 \pm 1.80$ & $9.21 \pm 1.56$ & $9.08 \pm 1.94$ & $8.66 \pm 2.05$ & 0.36 \\
\hline Utilidad como tutorial & $9.03 \pm 1.86$ & $9.15 \pm 1.62$ & $9.07 \pm 1.99$ & $8.66 \pm 2.17$ & 0.65 \\
\hline Probabilidad de futura consulta & $8.63 \pm 2.18$ & $8.80 \pm 1.96$ & $8.75 \pm 2.07$ & $7.96 \pm 2.80$ & 0.70 \\
\hline Probabilidad de recomendarlo & $8.71 \pm 2.10$ & $8.86 \pm 1.76$ & $8.85 \pm 2.03$ & $8.06 \pm 2.83$ & 0.39 \\
\hline
\end{tabular}

Los análisis de correlación de Spearman entre los factores de usabilidad con el promedio escolar y la percepción de la experiencia en las clases de metodología de la investigación solamente arrojó tres correlaciones positivas modestas entre el promedio académico de los estudiantes con la facilidad de acceso de la infografía $(\mathrm{Sr}=0.16$; $\mathrm{p}<.05)$, con su atractivo visual $(\mathrm{Sr}=0.20 ; \mathrm{p}<.05)$ y con la facilidad de acceso a la presentación $(\mathrm{Sr}=0.21 ; \mathrm{p}<.05)$. Los alumnos con mejor promedio tendieron a calificar mejor estos aspectos, aunque la asociación es pequeña. 
Respecto a si los estudiantes se consideraban capaces de explicar el contenido de los recursos después de interactuar con ellos la Tabla 5 muestra diferencias significativas $(p<.01)$ en las evaluaciones que se hicieron para explicar la información que contenían. Las diferencias más grandes están en la evaluación de la presentación. Solo el 33\% se sintió capaz de explicarla comparado con el 74.8 y el 73.6 de los que se sintieron capaces de explicar la infografía y el vídeo respectivamente. En el mismo sentido, el $11 \%$ de los que revisaron la presentación no se sintieron capaces de explicar su contenido, comparados con el $0.6 \%$ y el casi $5 \%$ que reportaron no poder hacerlo para la infografía y el vídeo, respectivamente.

\section{Tabla 5}

Capacidad percibida para explicar la información de un recurso, $n(\%)$

\begin{tabular}{lccc}
\hline Tipo de recurso & Si & Si, pero con ayuda & No \\
\hline Infografia & $122(74.9)$ & $40(24.5)$ & $1(0.6)$ \\
Presentación & $54(33.1)$ & $91(55.8)$ & $18(11.0)$ \\
Video & $120(73.6)$ & $39(23.9)$ & $4(4.7)$ \\
\hline
\end{tabular}

$\chi_{(4)}^{2}=83.0 ; p=0.00$

\section{DISCUSIÓN Y CONCLUSIONES}

El objetivo de esta investigación fue evaluar la usabilidad de recursos TIC para apoyar el aprendizaje de metodología de la investigación en alumnos de psicología, desde la perspectiva de los alumnos. De forma general tanto las infografías como los vídeos fueron los recursos mejor evaluados. La presentación de power point fue el recurso que recibió más recomendaciones de mejora.

Al considerar de forma general los factores académicos como el promedio, semestre y experiencia en la materia de metodología de la investigación resulta interesante observar cómo al parecer en la muestra evaluada la valoración de la experiencia en metodología de la investigación se va reduciendo conforme a los alumnos avanzan de semestre, lo cual coincide 
con la investigación de Montero (2019) quien reporta que las experiencias de los estudiantes decaen debido a las estrategias de enseñanza de los profesores. Esto refleja la percepción de los alumnos respecto a la cantidad de conocimiento en el área que tiene el docente y si este no se ve evidenciado llega a desmotivar al alumno. Incluso modificando su percepción sobre cuál es el objetivo de la clase de metodología de la investigación, ya que consideran que solo deben hacer un proyecto de investigación lo cual resulta en una sobrecarga de trabajo que en algunos casos sucede desde el primer semestre, generando bajas expectativas de aprendizaje y quizá en consecuencia experiencias negativas.

Con relación a las experiencias de estudiantes de etapa básica de la carrera de psicología en la FES Z, particularmente en la unidad de aprendizaje de métodos y técnicas de investigación sobre los aspectos o técnicas implementan sus profesores al momento de estar en el curso, Contreras (2016) reportó que los profesores no implementan recursos TIC al momento de desarrollar su curso.

Respecto a la evaluación de usabilidad de forma general esta fue valorada globalmente de forma positiva con especial énfasis en los recursos de video y menos en la presentación, este fue el recurso que requiere una mayor revisión y mejoría. Asimismo, parece que la forma de medir la usabilidad a través de la capacidad percibida para explicar el contenido de los recursos resultó ser una forma apropiada de evaluar un aspecto de la utilidad de los materiales.

Un dato que llama la atención es la ausencia de diferencias estadísticamente significativas en las valoraciones de los aspectos de utilidad de la información, utilidad del recurso como tutorial o probabilidad de futura 
consulta entre los semestres. Hipotéticamente, se hubiera esperado una evaluación diferencial a medida que aumentaba el semestre, sin embargo, la falta de diferencias podría reflejar un conocimiento (o desconocimiento) uniforme de los temas lo que podría sugerir una nula o mínima ganancia de conocimiento a medida que se avanza en la carrera.

Es en ese punto donde pareciera adecuado implementar estos recursos TIC u otros recursos gráficos y analizar cómo podrían influir en la construcción de la percepción de la unidad de aprendizaje, que experiencias se crean y que nivel de conocimiento desarrollan los alumnos.

La principal limitación del estudio radica en la generalización de los resultados. Por una parte, aunque participaron alumnos de los 4 semestres en activo al momento de la realización del estudio, la proporción de participantes de tercer semestre fue muy baja y, por la otra, al tratarse de una muestra autoseleccionada no representativa, la validez externa del estudio está comprometida. No obstante, los resultados parecen sugerir que en principio los recursos evaluados pueden ser potencialmente útiles como apoyo para la enseñanza de la unidad de aprendizaje e indicaron oportunidades de mejora. Además de estas posibilidades de mejora, la amplitud de temas que comprende la metodología de la investigación invita a continuar desarrollando y evaluando recursos que pudieran tener el mismo propósito. Otro aspecto por revisar es el contenido de los recursos. Si bien fueron desarrollados con la supervisión de profesores e investigadores que imparten la actividad de seminario de investigación, conviene someter a evaluación los aspectos particulares que se incluyen en los recursos. 
Finalmente, es importante mencionar que los recursos son únicamente un complemento o una herramienta para mejorar el proceso de enseñanza aprendizaje, no un sustituto para el docente. Así mismo las experiencias de los alumnos y el cumplimiento de los propósitos de la unidad de aprendizaje son situaciones multifactoriales. Es decir, aún hay mucho que explorar para optimizar todo el proceso de enseñanza de metodología de la investigación y en consecuencia el desarrollo de una habilidad que puede ser determinante para el desarrollo profesional del psicólogo.

\section{REFERENCIAS}

Araujo, D., \& Bermúdez, J. (2009). Limitaciones de las tecnologías de información y comunicación en la educación universitaria. Horizontes Educacionales, 14(1), 9-24.

Ayala, R., Mesinga, H., Labbé, C., \& Obando, I. (2010). Congruencia entre el diseño curricular y la evaluación de los aprendizajes esperados en cátedras impartidas en una universidad chilena. Estudios pedagógicos, 36(1), 53-67.

Behar, D. (2008). Metodología de la investigación. Shalom.

Behrens, J., Van-Elzakker, C., \& Schmidt, M. (2015). Testing the Usability of OpenStreetMap's iD Tool. Cartographic Journal, 52(2), 177-184.

Cacheiro, M. (2011). Recursos educativos tic de información, colaboración y aprendizaje. Pixel-Bit. Revista de Medios y Educación, 39, 69-81.

Contreras, M. (2016). La enseñanza de la psicología. UNAM, FES Zaragoza.

David, D., Cristea, I., \& Hofmann, S. (2018). Why Cognitive Behavioral Therapy Is the Current Gold Standard of Psychotherapy. Frontiers in Psychiatry, 9, 4.

Díaz, R. (1971). La enseñanza de la investigación en psicología en Iberoamérica: Un paradigma. Revista Latinoamericana de Psicología, 3(1),5-36. 
Fernández, C., Baptista, P., Hernández-Sampieri, R. (2014). Metodología de la Investigación. McGraw Hill: México

FES Zaragoza. (2010). Propuesta de modificación al plan y programas de estudio de la licenciatura en psicología (sistema presencial). Disponible en: https://www.zaragoza.unam.mx/wp-content/ portalfesz2019/Licenciaturas/ psicologia/plan_estudios_psicologia. pdf

Frege, T. (2011). Usabilidad web. Acta Nova, 5(1), 138-160.

Gómez, S. (2012). Metodología de la investigación. México: Red Tercer Milenio.

Hernández, V. (2019). Gestión 2018-2022 (Primer informe). UNAM, FES Zaragoza. Disponible en https://www.zaragoza.unam.mx/ wp-content/Porta12015/InformesGestion/archivos_gestion/ PrimerInforme_Actividades2018-2022.pdf

Ibarra, P. (2017). La titulación por tesis en México: el problema de su conceptualización. Revista Dilemas Contemporáneos: Educación, Política y Valores, 31(3), 17.

INEGI. (2020). Encuesta Nacional sobre Disponibilidad y Uso de Tecnologías de la Información en los Hogares (ENDUTIH) (2019). Disponible en: https://www.inegi.org.mx/contenidos/saladeprensa/ boletines/2020/OtrTemEcon/ENDUTIH_2019.pdf

Jiménez Pérez, A. L., Vargas Contreras, E., \& Martínez Martínez, K. I. (2020). Disposición a la práctica basada en evidencia: un estudio con alumnos de Psicología. Enseñanza e Investigación en Psicología, 2(3), 328-337.

Landa, E., Martínez, A., \& Sánchez, J. (2013). Medicina basada en la evidencia y su importancia en la medicina conductual. Psicología y Salud, 23, 273-282.

López, M. (2007). Uso de las TIC en la educación superior de México: Un estudio de caso. Apertura, 7(7), 63-81. 
Merrill, D.M. (2006) Levels of instructional strategy. Educational Technology, 46(4).

Montero, G. (2019). Experiencias en el proceso de enseñanza-aprendizaje de la Metodología de Investigación en estudiantes de psicología de pregrado: Análisis de grupos focales. [Tesis para obtener el grado de licenciada en psicología, Universidad Nacional Autónoma de México]

Nielsen, J. (1992). The usability engineering life cycle. Computer, 25(3), 12-22. https://doi.org/10.1109/2.121503

Pablos-Pons, J., \& Cabero-Almenara, J. (1990). El video en el aula. El video como mediador del aprendizaje. Revista de educación, 291, 351-370.

Quant, D., \& Trujillo, S. (2014). Psicología clínica basada en la evidencia y su impacto en la formación profesional, la investigación y la práctica clínica. Revista Costarricense de Psicología, 33(2), 123-136.

Rodríguez, N., Mendoza, M., \& Ferrer, L. (2014). Influencia de las Tecnologías de Información y Comunicación en los roles e interrelaciones entre estudiantes y docentes en programas presenciales de educación superior. Hallazgos, 22(11), 435-454.

Sánchez, R. (2014). Enseñar a investigar: Una didáctica nueva de la investigación en ciencias Sociales y humanas (4a ed.). Ciudad de México: UNAM.

Sáenz, M. (2010). Utilización de las TIC en el proceso de enseñanza aprendizaje, valorando la incidencia real de las tecnologías en la práctica docente. Revista Docencia e Investigación, 35(20), 183-204.

Sociedad Mexicana de Psicología (2007). Código ético del Psicólogo. Trillas.

Universidad Nacional Autónoma de México (UNAM). (2018). Oferta académica. Disponible en http://oferta.unam.mx/indice-alfabetico. html 
UNAM. (2020). Agenda Estadística UNAM 2020. Planeación UNAM. https://www.planeacion.unam.mx/Agenda/2020/disco/

Vega, O. (2016). De las TIC en la educación a las TIC para la educación. Vector, 11, 24-29.

Envío a dictamen: 1 de junio de 2021

Reenvío: 8 de junio de 2021

Aprobación:17 de junio de 2021

Daniela Jacqueline Vera Solis. Lic. En Psicología por la Facultad de Estudios Superiores Zaragoza. Ha participado en líneas de investigación relacionadas a la enseñanza de metodología de la investigación a estudiantes de psicología, con experiencia en el desarrollo de recursos electrónicos realizados con base en el diseño instruccional. Correoe: dvera2143@gmail.com

Ángel Francisco García Pacheco. Profesor de Tiempo Completo adscrito al área de Psicología Clínica y de la Salud de la FES Zaragoza, Dr.(c) en Ciencias de la Salud Mental Pública por la Facultad de Medicina de la UNAM, Mtro. en Psicología con especialidad en Medicina conductual por la Facultad de Psicología de la UNAM y Lic. en Psicología por la Facultad de Estudios Superiores Iztacala de la UNAM. Pertenece a la línea de investigación sobre Psicología de la Salud en la división de investigación de la FES Zaragoza. También está interesado en la investigación de la enseñanza de la Psicología en entornos presenciales y virtuales. Responsable y corresponsable de proyectos de investigación financiados en las líneas de trabajo mencionadas. Correo-e: angel.garcia@zaragoza.unam.mx 
Karina Serrano Alvarado. Profesora adscrita al área de Psicología Clínica y de la Salud de la FES Zaragoza, Dra.(c) en Ciencias de la Salud con concentración en epidemiología por la Facultad de Medicina de la UNAM, Mtra. en Psicología con especialidad en Medicina Conductual por la Facultad de Psicología de la UNAM y Lic. en Psicología por la FES Zaragoza, UNAM. Pertenece a la línea de investigación sobre Psicología de la Salud en la división de investigación de la FES Zaragoza. Colaboradora en diversos proyectos con financiamiento sobre temas relacionados con la promoción de las conductas de salud y la enseñanza de la psicología. Correo-e: karina.serrano@zaragoza.unam.mx Mario E. Rojas Russell. Profesor Titular de Tiempo Completo en el área de Psicología Clínica y de la Salud de la FES Zaragoza. Es Dr. en Ciencias de Salud-Epidemiología por la Facultad de Medicina de la UNAM. Maestro en Ciencias de la Salud-Epidemiología por la Escuela de Salud Pública de México del INSP y Lic. En Psicología por la ENEP Zaragoza. Tutor del Programa de Maestría y Doctorado en Ciencias Médicas, Odontológicas y de la Salud y del Posgrado en Psicología, ambos de la UNAM. Responsable de proyectos de investigación y enseñanza en los campos de Psicología y Salud, Epidemiología, Salud Pública, Promoción de la Salud y Enseñanza de la Metodología de la Investigación. Correo-e: mario.rojas@zaragoza.unam.mx 